\title{
Does GP training in depression care affect patient outcome? - A systematic review and meta- analysis
}

Claudia Sikorski ${ }^{1,2,5^{*}}$, Melanie Luppa ${ }^{1}$, Hans-Helmut König ${ }^{3}$, Hendrik van den Bussche ${ }^{4}$ and Steffi G Riedel-Heller ${ }^{1}$

\begin{abstract}
Background: Primary care practices provide a gate-keeping function in many health care systems. Since depressive disorders are highly prevalent in primary care settings, reliable detection and diagnoses are a first step to enhance depression care for patients. Provider training is a self-evident approach to enhance detection, diagnoses and treatment options and might even lead to improved patient outcomes.

Methods: A systematic literature search was conducted reviewing research studies providing training of general practitioners, published from 1999 until May 2011, available on the electronic databases Medline, Web of Science, PsycINFO and the Cochrane Library as well as national guidelines and health technology assessments (HTA).

Results: 108 articles were fully assessed and 11 articles met the inclusion criteria and were included. Training of providers alone (even in a specific interventional method) did not result in improved patient outcomes. The additional implementation of guidelines and the use of more complex interventions in primary care yield a significant reduction in depressive symptomatology. The number of studies examining sole provider training is limited, and studies include different patient samples (new on-set cases vs. chronically depressed patients), which reduce comparability.

Conclusions: This is the first overview of randomized controlled trials introducing GP training for depression care. Provider training by itself does not seem to improve depression care; however, if combined with additional guidelines implementation, results are promising for new-onset depression patient samples. Additional organizational structure changes in form of collaborative care models are more likely to show effects on depression care.
\end{abstract}

Keywords: depression, primary care, training, health service

\section{Background}

Depressive disorders are highly prevalent in the general public. The 12-month prevalence of Major Depression among Europeans has shown to be approximately 6.9\% while conservative estimates of the lifetime prevalence range up to $14 \%[1,2]$. Depression is associated with significant functional impairment and reduced quality of life [3,4], excess mortality rates [5] and particularly high costs for society and health care systems [6-9]. Considering the large effects of the disease on individuals and

\footnotetext{
* Correspondence: Claudia.Sikorski@medizin.uni-leipzig.de ${ }^{1}$ Institute of Social Medicine, Occupational Health and Public Health, University of Leipzig, Leipzig, Germany

Full list of author information is available at the end of the article
}

society, it seems clear that early detection and treatment is a desirable goal in order to promote remission and reduce negative consequences [10].

While 50 to 70 per cent of all depressed patients consult their primary care physician during an episode, therefore making them the profession most likely to be seen $[11,12]$, depression in primary care remains underrecognised and under-treated [13]. As Bijl and colleagues (2004) summarise, the elements of detection, diagnosis and treatment determine successful depression management in health care. Previous trials showed that approximately 50 per cent of all depressed patients in primary care were not diagnosed as such [14-16]. This is a major downfall in depression care, since even
C Biomed Central 
subthreshold depression episodes may be clinically significant [17]. Obviously part of this is due to reluctance to seek treatment among patients themselves, resulting from concerns on effectiveness of treatment and perceived absence of treatment necessity [18].

To objectify diagnoses, the use of screening instruments has been promoted by the U.S. Preventive Services Task Force, when adequate treatment and care possibilities are available [19]. Reviews showed that screening alone does not improve depression outcomes for patients [20], but needs further organizational changes [21]. These structural interventions, including collaborative care approaches as well as provider training, represent an attempt to increase detection and diagnosis of depressed patients and therefore promote enhanced treatment. Several treatment options that support primary care physicians in treatment and that are also directly delivered by general practitioners (such as PST - problem solving therapy) have been found to be effective for depression [22,23].

Primary care practices play a central role in many health care systems- this kind of gate-keeping is even associated with improved coordination and outcomes [24]. This circumstance makes general practitioners ideal as a base for first steps in treatment, also referred to as a "stepped care" approach [25]. "Watchful waiting" and low intense interventions such as self-help approaches have to be encouraged as useful strategies [26]. In order to make full use of this opportunity, improvement of detection rates and diagnosis is inevitable. Improving skills of primary care providers can be achieved by different strategies. Consultation-liaison involves a persistent educational supervision of the general practitioner by a mental health specialist. This approach has not been shown to be effective in reducing depressive symptoms [27]. As indicated by Cape et al. (2010), another point of intervention can be to train primary care providers in diagnosis and treatment strategies without the inclusion of mental health specialists (such as collaborative care), considering limited financial resources of health care systems [27]. Moderated by higher detection rates and better knowledge on treatment options, improvement on this level could subsequently lead to higher remission rates in less time and improved depression outcomes.

In the past, these programmes of provider education have been evaluated, yielding unclear results on effectiveness of the intervention regarding health gain outcomes [21]. This study therefore reviews current literature for an updated overview. It is the first overview of randomized controlled trials that exclusively investigates interventions that apply practitioner training.

\section{Methods}

\section{Literature Search}

This review was prepared according to the systematic literature review guidelines of the Centre for Reviews and Dissemination [28] and follows PRISMA (Preferred Reporting Items for Systematic reviews and Meta-Analyses) suggestions [29]. A systematic literature search was conducted reviewing research studies, published from 1999 until May 2011, available on the electronic databases Medline, Web of Science, PsycINFO and the Cochrane Library as well as national guidelines and health technology assessments (HTA). In addition, the bibliographies of the selected articles were searched. Grey literature was not searched. 1999 as a starting point of the search was chosen to include at least the last 10 years of publications. The latest review on this topic, including studies from 1999 onward, was conducted in 2003, and we meant to include those studies as well [21]. The aim was to evaluate if newer, more recent studies would show clearer effects of the intervention than previous overviews.

The terms (depression OR depressive disorder) AND (general practitioner OR general practice OR primary care OR family practice) AND (training OR education) served as search criteria within titles and abstracts. All terms were also used as MeSH terms where applicable. Test searches were run preceding the actual search in order to determine the right search terms. Additional File 1 shows the Medline search strategy in detail. The search was limited to English and German language publications.

\section{Inclusion criteria}

Abstracts were screened by two authors using the following inclusion criteria: (i) randomized controlled trials (RCT) or review articles (ii) of the adult ( $\geq 18$ years) general population, (iii) evaluating interventional programmes including general practitioner training, mentioned in the abstract and (iv) reporting effects on depressive symptomatology. All extracted review articles were scanned and hand-searched for further relevant publications from 1999 onward.

Studies examining effects in specific study samples (such as diabetic patients with co-morbid depression) were excluded. Research of those specific samples was thought to provide only limited evidence for primary health care patients in general. All articles where a clear decision could not be made based on title or abstract were retrieved for a more detailed analysis. In case of disagreement, a third reviewer was consulted and then a consensus decision was achieved.

Training and education of general practitioners was defined as a professional intervention [21] that involves 
the use of guidelines or short training classes with a focus on optimising diagnosis as well as treatment. Studies involving additional organisational interventions were excluded. Additional file 2 gives an overview on excluded studies.

\section{Data extraction}

Primarily, methodical data on sampling, study design, intervention procedure, and outcome criteria were extracted from all selected studies. Extraction of results focussed on assessing symptom alteration primarily. Only data related to a change in symptom severity (scale scores, remission rates) were extracted. Effect sizes were only calculated for the outcomes considered as relevant (symptom change). Secondly, the selection criteria described in the above section were then reapplied to ensure accurate study inclusion.

\section{Study Quality}

Study quality was also assessed by two independent raters using a modified scale based on work by Moncrieff and colleagues [30]. The scale was modified by leaving out irrelevant items such as medication sideeffects. It consists of 21 items leading to a maximum score of 42 points (Table 1). Each item, if not specified otherwise, was scored as 2 (fully met the quality criterion), 1 (partially met the quality criterion) or as 0 (did not meet the quality criterion). After a first independent run-through of ratings, the two raters met with a third independent researcher in order to discuss disagreements in scoring until a consensus was reached. Study protocols were consulted where possible.

\section{Effect Size and meta-analysis}

Whenever applicable, standardized mean effect sizes (Cohen's d) were calculated from the data reported. At times, studies only reported scores that could not be used in effect size calculation and efforts to retrieve data directly from the authors were made. Data was entered to interpret negative standardized means in favour of the intervention. Results of cluster trials were used when the authors accounted for the effect of cluster randomization properly. According to Cohen (1988), effect sizes of 0.2 are considered small, while $d=0.5$ represents a moderate effect and $d=0.8$ is regarded a large effect [31]. A meta-analysis was conducted using Review Manager Software [32]. Due to the diversity of GP training in the studies, standardized mean effects were pooled - firstly, for studies with GP training only, secondly, for studies introducing additional guidelines and lastly for studies including more complex interventions. Subgroup analysis (patient inclusion, age of patients) were not carried out due to the unavailability of sufficient data. Analysis of the heterogeneity of prevalence across studies was done through $\mathrm{I}^{2}$ statistic and Cochran Q. A fixed effect model was applied since heterogeneity was low.

\section{Results \\ Search results}

The results of the systematic literature search are shown in Figure 1. Interrater reliability showed substantial agreement $($ Kappa $=0.74)$. Overall, 108 potentially relevant articles were identified. After retrieving all full articles, 97 further articles were rejected as not fulfilling the selection criteria. Eleven articles were assessed and included for detailed analysis. Relevant study characteristics can be found in Table 2. Three articles are double publications of the same studies and will be subtracted for the following overview. The QuEST intervention is described in detail in a publication by Rost et al. (2000). Therefore, this reference will be used when referring to that study.

\section{Study characteristics}

All, but one, studies were conducted in anglophone countries, among them Great Britain $(n=4)$ and one study each in Canada, the United States and Australia. The sample sizes varied from 145 [33] to 733 [34,35]. Three studies included patients with a categorical (e.g. diagnostic system based) diagnosis of depression $[33,36,37]$, while the other five made use of symptom rating scales (self-report scales). Gask et al. (2004) and Worrall et al. (1999) both based their samples on referrals by the general practitioner (having the GP determine whether the patient was depressed) but applied dimensional instruments to ensure accuracy of diagnoses [38,39]. All but one study used a cluster allocation design, randomising all included general practices to either intervention or control group. Only LlewellynJones et al. (1999) conducted a serial designed survey, randomising each consecutive patient to the experimental groups [40]. Four research groups planned to train the control group practitioners after the end of the trial while the other four had them assigned to usual care groups with no further support provided. However, three study teams chose to provide the physicians of the control groups with depression specific guidelines $[36,39,40]$. In the Dutch study it is highlighted, that all practitioners are generally encouraged to adhere to guideline concordant treatment [33].

\section{Interventions}

As for interventional strategies, education and training of the participating general practitioners was the sole intervention in three studies $[33,38,41]$. These studies did not provide any other organisational support for the practices. King et al. (2002) pose an exception to the 
Table 1 Quality assessment (Based on Moncrieff et al., 2001)

\begin{tabular}{|c|c|}
\hline Criterion & Score and rating criteria \\
\hline $\begin{array}{l}\text { (1) Objectives and specification } \\
\text { main outcomes a priori }\end{array}$ & $\begin{array}{l}0=\text { objectives unclear } \\
1=\text { objectives clear but main outcomes not specified a priori } \\
2=\text { objectives clear with a priori specification of main method } \\
\text { for assessment of outcome }\end{array}$ \\
\hline (2) Adequate sample size (n per group) & $\begin{array}{l}0=\text { inadequate }(<50 / \text { group }) \\
1=\text { moderate }(50-100 / \text { group }) \\
2=\text { large }(>100 / \text { group or justified by power calculations })\end{array}$ \\
\hline (3) Appropriate duration of trial including follow up & $\begin{array}{l}0=\text { too short }(<3 \text { months) } \\
1=\text { reasonable length }(3-6 \text { months) } \\
2=\text { long enough for assessment of long term outcomes (6-12 months) }\end{array}$ \\
\hline (4) Power calculation & $\begin{array}{l}0=\text { not reported } \\
1=\text { mentioned without details } \\
2=\text { details of calculations provided }\end{array}$ \\
\hline (5) Method of allocation & $\begin{array}{l}0=\text { unrandomized and likely to be biased } \\
1=\text { partially or quasi randomized with some bias possible } \\
2=\text { randomized allocation }\end{array}$ \\
\hline (6) Concealment of allocation & $\begin{array}{l}0=\text { not done or not reported } \\
2=\text { concealment of allocation code detailed }\end{array}$ \\
\hline $\begin{array}{l}\text { (7) Clear description of treatments (including doses of drugs used) and } \\
\text { adjunctive treatments }\end{array}$ & $\begin{array}{l}0=\text { main treatments not clearly described } \\
1=\text { inadequate details of main or adjunctive treatments } \\
2=\text { full details of main and adjunctive treatments }\end{array}$ \\
\hline (8) Blinding of subjects & $\begin{array}{l}0=\text { not done } \\
1=\text { done but no test of blind } \\
2=\text { done and integrity of blind tested }\end{array}$ \\
\hline (9) Source of subjects described and representative sample recruitment & $\begin{array}{l}0=\text { source of subjects not described } \\
1=\text { source of subjects given but no information on sampling or use } \\
\text { of unrepresentative sample (for example, volunteers) } \\
2=\text { source of subjects described plus representative sample taken } \\
\text { (for example, all consecutive admissions or referrals, or random } \\
\text { sample taken) }\end{array}$ \\
\hline (10) Use of diagnostic criteria (or clear specification of inclusion criteria) & $\begin{array}{l}0=\text { none } \\
1=\text { diagnostic criteria or clear inclusion criteria } \\
2=\text { diagnostic criteria plus specification of severity }\end{array}$ \\
\hline $\begin{array}{l}\text { (11) Record of exclusion criteria and number of exclusions and refusals } \\
\text { reported }\end{array}$ & $\begin{array}{l}0=\text { criteria and number not reported } \\
1=\text { criteria or number of exclusions and refusals not reported } \\
2=\text { criteria and number of exclusions and refusals reported }\end{array}$ \\
\hline (12) Description of sample demographics & $\begin{array}{l}0=\text { little/no information (only age/sex) } \\
1=\text { basic details (for example, marital status/ethnicity) } \\
2=\text { full description (for example, socioeconomic status, } \\
\text { clinical history) }\end{array}$ \\
\hline (13) Blinding of assessor & $\begin{array}{l}0=\text { not done } \\
1=\text { done but no test of blind } \\
2=\text { done and integrity of blind tested }\end{array}$ \\
\hline (14) Record of number and reasons for withdrawal by group & $\begin{array}{l}0=\text { no info on withdrawals by group } \\
1=\text { withdrawals by group reported without reason } \\
2=\text { withdrawals and reason by group }\end{array}$ \\
\hline $\begin{array}{l}\text { (15) Outcome measures described clearly (and therefore replicable) or use } \\
\text { of validated (or referenced) instruments }\end{array}$ & $\begin{array}{l}0=\text { main outcomes not described clearly } \\
1=\text { some of main outcomes not clearly described } 2=\text { main outcomes } \\
\text { clearly described or valid and reliable } \\
\text { instruments used }\end{array}$ \\
\hline $\begin{array}{l}\text { (16) Information on comparability and adjustment } \\
\text { for differences in analysis }\end{array}$ & $\begin{array}{l}0=\text { no information on comparability } \\
1=\text { some information on comparability with appropriate } \\
\text { adjustment } \\
2=\text { sufficient information on comparability with appropriate } \\
\text { adjustment }\end{array}$ \\
\hline (17) Inclusion of all subjects in analyses (Intention to treat analysis) & $\begin{array}{l}0=\text { no } \\
2=\text { yes }\end{array}$ \\
\hline $\begin{array}{l}\text { (18) Presentation of results with inclusion of data forre-analysis of main } \\
\text { outcomes (for example, SDs) }\end{array}$ & $\begin{array}{l}0=\text { little information presented } \\
1=\text { adequate information } \\
2=\text { comprehensive }\end{array}$ \\
\hline
\end{tabular}


Table 1 Quality assessment (Based on Moncrieff et al., 2001) (Continued)

\begin{tabular}{ll}
\hline (19) Appropriate statistical analysis (including correction for multiple tests & $0=$ inadequate \\
where applicable) & $1=$ adequate \\
& $2=$ comprehensive and appropriate \\
\hline (20) Conclusions justified & $0=$ no \\
$1=$ partially \\
$2=$ yes \\
\hline $\begin{array}{l}0=\text { no } \\
21) \text { Declaration of interests (for example, } 0=\text { no }\end{array}$ \\
source of funding)
\end{tabular}

other studies, since physicians here are trained to provide a specific interventional method (brief cognitive behavioural therapy) [41], while in the remaining trial physicians were only provided with lectures on assessment and treatment of depression. Four studies made use of guideline implementation [35-37,39]. These studies can be seen as providing a more intense intervention, as practitioners were trained and additionally

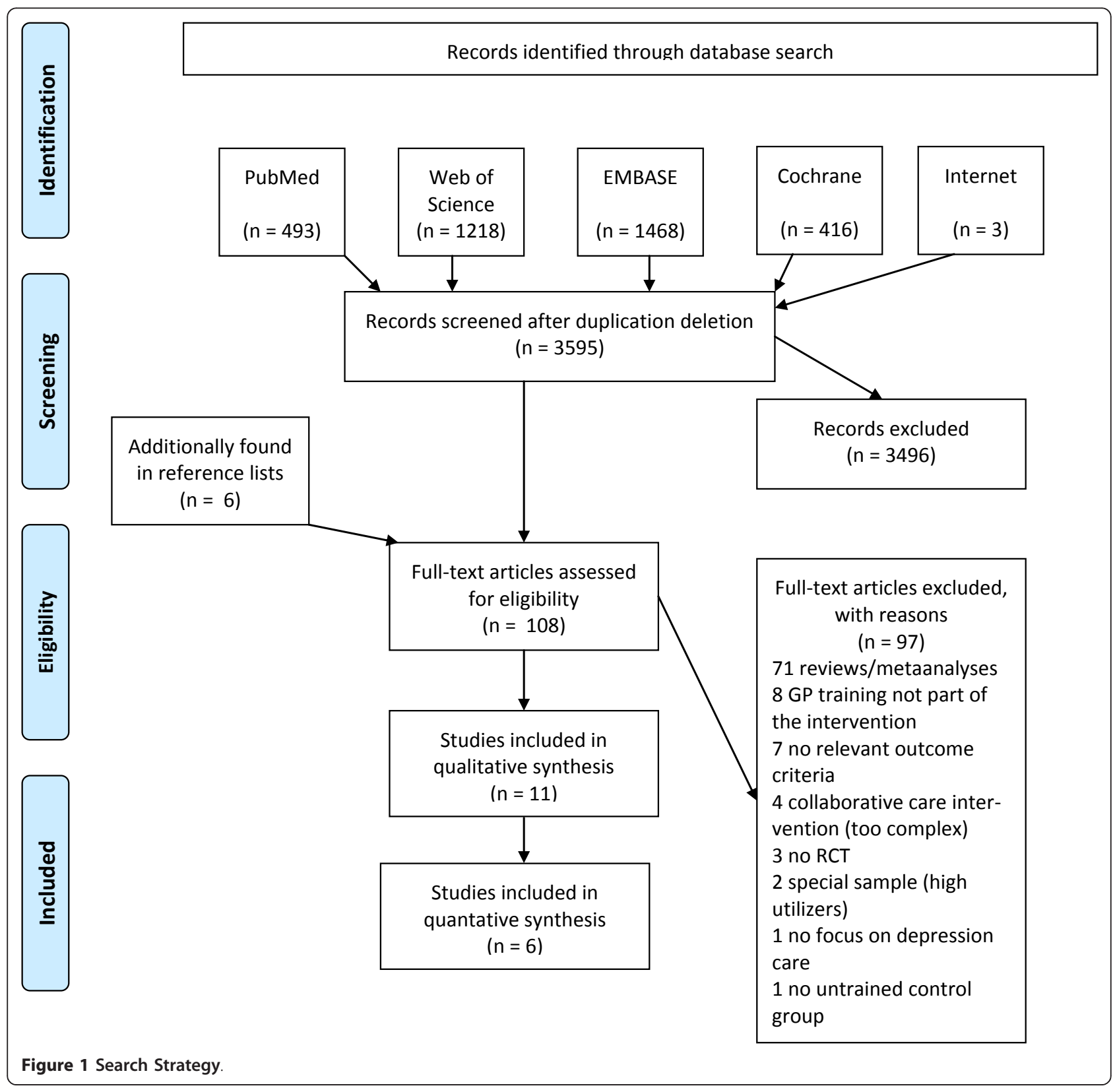


Table 2 Study Characteristics

\begin{tabular}{|c|c|c|c|c|c|c|c|c|}
\hline Study & Country & $\begin{array}{l}\text { Recruitment, } \\
\text { Inclusion Criteria }\end{array}$ & Randomization & $\mathrm{N}^{\mathrm{a}}$ & Intervention, Role of GP Training & $\begin{array}{l}\text { Control } \\
\text { Group }\end{array}$ & Comparison & Quality \\
\hline $\begin{array}{l}\text { Baker } \\
(2001) \text { [36] }\end{array}$ & GB & $\begin{array}{l}\text { Consecutive patients; } \\
\geq 18 \text { yrs } \\
\text { Patients seeking } \\
\text { consultation for new- } \\
\text { onset depression }\end{array}$ & Practices & 402 & $\begin{array}{l}\text { Tailored intervention to promote } \\
\text { guideline implementation } \\
\text { (additional feedback, educational } \\
\text { visits, group discussions) } \\
\text { Additional to guideline }\end{array}$ & $U C^{b}$ & $\begin{array}{l}\text { Patients of } \\
\text { experimental group } \\
\text { vs. control group }\end{array}$ & 30 \\
\hline $\begin{array}{l}\text { Bosmans } \\
\text { (2006) [33] }\end{array}$ & $\mathrm{NL}$ & $\begin{array}{l}\text { Consecutive patients; } \\
\geq 55 \text { yrs } \\
\text { PRIME-MD = MD }\end{array}$ & Practices & 145 & $\begin{array}{l}4 \text { hrs training session on screening, } \\
\text { diagnosis and treatment as in } \\
\text { Dutch guidelines }\end{array}$ & UC & $\begin{array}{l}\text { Patients of } \\
\text { experimental group } \\
\text { vs. control group }\end{array}$ & 39 \\
\hline $\begin{array}{l}\text { Gask (2004) } \\
{[38]}\end{array}$ & $\mathrm{GB}$ & $\begin{array}{l}\text { GP referral; } \\
16-65 \text { yrs } \\
\text { Intention or current } \\
\text { treatment of } \\
\text { depression } \\
\text { (symptoms < } 6 \text { mo) } \\
\text { HAM-D } \geq 13\end{array}$ & Practice & 189 & $\begin{array}{l}\text { Acquisition of clinical skills, } 5 \\
\text { lectures à } 2 \text { hrs on assessment and } \\
\text { treatment; } \\
\text { Sole intervention }\end{array}$ & WL & $\begin{array}{l}\text { Patients of } \\
\text { experimental group } \\
\text { vs. control group }\end{array}$ & 36 \\
\hline $\begin{array}{l}\text { Kendrick } \\
\text { (2001) [34] } \\
\text { Thompson } \\
\text { (2000) [35] }\end{array}$ & GB & $\begin{array}{l}\text { Consecutive patients; } \\
\geq 16 \text { yrs } \\
\text { HADS-D } \geq 8\end{array}$ & Practice & 733 & $\begin{array}{l}\text { Guideline implementation \& GP } \\
\text { training ( } 4 \text { h seminars, educators } \\
\text { available for } 9 \text { more mo); } \\
\text { Additional to guideline }\end{array}$ & $\mathrm{WL}$ & $\begin{array}{l}\text { Patients of } \\
\text { experimental group } \\
\text { vs. control group } \\
\text { Sensitivity of } \\
\text { recognition rates of } \\
\text { experimental group } \\
\text { vs. control group }\end{array}$ & 36 \\
\hline $\begin{array}{l}\text { King (2002) } \\
{[41]}\end{array}$ & $\mathrm{GB}$ & $\begin{array}{l}\text { Consecutive patients; } \\
\geq 18 \mathrm{yrs} \\
\text { HADS-D/A } \geq 11\end{array}$ & Practice & 272 & $\begin{array}{l}\text { Training of GPs in brief cognitive } \\
\text { behaviour therapy ( } 4 \text { half day } \\
\text { workshops); } \\
\text { Sole intervention }\end{array}$ & WL & $\begin{array}{l}\text { Patients of } \\
\text { experimental group } \\
\text { vs. control group }\end{array}$ & 34 \\
\hline $\begin{array}{l}\text { Llewellyn- } \\
\text { Jones } \\
\text { (1999) } \\
{[40]}\end{array}$ & AUS & $\begin{array}{l}\text { Residential facility; } \\
\geq 65 \text { yrs } \\
\text { GDS } \geq 10 \\
\text { MMSE } \geq 18\end{array}$ & Patient & 220 & $\begin{array}{l}\text { Shared Care Intervention, including } \\
\text { GP training \& education, health } \\
\text { education and promotion, } \\
\text { psychoeducation; } \\
\text { Central part of complex } \\
\text { intervention }\end{array}$ & $W L^{c}$ & $\begin{array}{l}\text { Experimental group } \\
\text { vs. control group }\end{array}$ & 33 \\
\hline $\begin{array}{l}\text { Rost (2001) } \\
{[44]} \\
\text { Pyne } \\
\text { (2003) [43] } \\
\text { Rost (2005) } \\
\text { [45] } \\
\end{array}$ & USA & $\begin{array}{l}\text { GP referral; } \\
\text { DSM III-R MD (latter } \\
\text { two studies exclude } \\
\text { patients currently in } \\
\text { treatment) }\end{array}$ & Practice & 479 & $\begin{array}{l}\text { QuEST intervention, } 4 \text { academic } \\
\text { telephone calls to implement } \\
\text { guidelines, nurse w/8-hour face-to- } \\
\text { face training; } \\
\text { Guidelines implementation }\end{array}$ & $U C$ & $\begin{array}{l}\text { Patients of } \\
\text { experimental group } \\
\text { vs. control group }\end{array}$ & 38 \\
\hline $\begin{array}{l}\text { Worrall } \\
\text { (1999) [39] }\end{array}$ & CAN & $\begin{array}{l}\text { GP referral; } \\
\text { GP diagnosis and } \\
\text { severity rating, later } \\
\text { CES-D } \geq 16\end{array}$ & Practice & 147 & $\begin{array}{l}\text { 3-hour sessions on guideline } \\
\text { implementation + possible } \\
\text { consultation of psychiatrist; } \\
\text { Guidelines implementation }\end{array}$ & $U C^{b}$ & $\begin{array}{l}\text { Patients of } \\
\text { experimental group } \\
\text { vs. control group }\end{array}$ & 26 \\
\hline
\end{tabular}

\footnotetext{
${ }^{a}$ At baseline; ${ }^{b}$ Receipt of guidelines by mail; ${ }^{\mathrm{c}}$ Assessment of control group during first period of study, followed by intervention and assessment of experimental group; ${ }^{d}$ Rost et al. (2001): comparison recently treated patients vs. patients beginning new treatment episode, analysis for both intervention groups.

Abbreviations: CES-D - Center for Epidemiologic Studies Depression Scale; CIDI - Composite International Diagnostic Interview; GDS - Geriatric Depression Scale; GP - General Practitioner; HADS-D/A - Hospital Anxiety and Depression Scale; HAM-D - Hamilton Depression Scale; MD - Major Depression; MMSE - Mini Mental Status Examination; mo - month(s); PRIME-MD - Primary care Evaluation of Mental Disorders; QI - Quality Improvement; UC - Usual Care; w/- with; WL - Waiting List; yrs - years.
}

received guidelines and guideline explanations. Rost et al. (2000) and Worrall et al. (1999) focussed the GP training on implementing guidelines [37,39]. Thompson et al. (2000) also educated practitioners but additionally tried to implement guideline concordant treatment [35]. Baker et al. (2001) used a tailored application of practitioner training by firstly analysing possible obstacles for successful guidelines implementation and then delivering individualised help to the GPs [36].
Regarding more complex interventions, it can be concluded that provider education plays the central part in the programme conducted by Llewellyn-Jones et al. (1999).

The mean quality score of all studies was at 34.91 points and ranged from 26 to 39 (individual scores in table 2). Criteria such as random allocation as proposed by the Cochrane Collaboration Handbook were adequately addressed by all studies [42]. 


\section{Effectiveness of provider training}

Table 3 summarises all study results. The three studies solely providing physician education found no change in symptom severity. Neither lectures for more qualified assessment and treatment [33,38], nor training in brief cognitive behavioural therapy [41] led to significant symptom change in patients of trained physicians. Introducing additional guidelines and using them during practitioner training, two studies showed a mid and long term significant change in symptom load $[39,43-45]$. Both trials report small effect sizes $(d=0.22$ -
0.29 ). Short term, one study was able to show an increases probability of reducing the depression score below a clinically relevant cut-point [36]. Yet, another study fails to show effects of the intervention introducing guidelines. Not only was there no effect of the practitioner training on diagnosis sensitivity and specificity, patient also do not profit symptom wise or regarding hospital admittance [34,35].

As for the more complex interventional strategies, there is one study in which general practitioner and provider training plays a central role [40]. In a sample of

Table 3 Study Results

\begin{tabular}{|c|c|c|c|c|c|c|}
\hline Study & $\begin{array}{l}\text { Follow } \\
\text { Up }\end{array}$ & $\begin{array}{l}\text { Attrition } \\
\text { Rate } \%^{a}\end{array}$ & Outcome & Results & Limitations & $\begin{array}{l}\text { Effect } \\
\text { Size }^{b}\end{array}$ \\
\hline $\begin{array}{l}\text { Baker } \\
\text { (2001) [36] }\end{array}$ & $\begin{array}{l}16 \\
\text { weeks }\end{array}$ & 6 & $\begin{array}{l}\text { Proportion of } \\
\text { patients W/ } \\
\mathrm{BDI}<11\end{array}$ & $\begin{array}{l}\text { Sign. diff in proportion of patients } w / B D I> \\
11(O R=2.5)\end{array}$ & $\begin{array}{l}\text { Tailored intervention that makes GP } \\
\text { comparison impossible since they all } \\
\text { received diff kinds of intervention }\end{array}$ & / \\
\hline $\begin{array}{l}\text { Bosmans } \\
\text { (2006) [33] }\end{array}$ & $12 \mathrm{mo}$ & 21 & PRIME-MD & No sign. diff in MD recovery & $\begin{array}{l}\text { Possible Hawthorne effect, less severe } \\
\text { episodes of MD in primary care, no } \\
\text { blinding of patients }\end{array}$ & -0.07 \\
\hline $\begin{array}{l}\text { Gask (2004) } \\
\text { [38] }\end{array}$ & $\begin{array}{l}3,12 \\
\mathrm{mo}\end{array}$ & 37 & HAM-D & $\begin{array}{l}\text { No sign. diff in scores at both follow up } \\
\text { points }\end{array}$ & $\begin{array}{l}\text { Use of a new-onset (depressed less than } 6 \\
\text { mo) sample } \\
\text { Attrition rate rather high }\end{array}$ & -0.24 \\
\hline $\begin{array}{l}\text { Kendrick } \\
\text { (2001) [34] }\end{array}$ & $12 \mathrm{mo}$ & 19 & $\begin{array}{l}\text { Hospital } \\
\text { Admittance }\end{array}$ & No sign. difference & $\begin{array}{l}\text { Implemented guidelines had been tested } \\
\text { in highly selected samples } \\
\text { Dimensional diagnosis } \\
\text { Potential conservative bias (chronic } \\
\text { depressed patients) }\end{array}$ & / \\
\hline \multirow[t]{2}{*}{$\begin{array}{l}\text { Thompson } \\
\text { (2000) [35] }\end{array}$} & $\begin{array}{l}6 \\
\text { weeks/ } \\
6 \text { mo }\end{array}$ & 50 & $\mathrm{HAD}$ & $\begin{array}{l}\text { No diff in improvement, no diff in caseness } \\
\text { rating at both points } \\
\text { Only patients recognized as cases at } \\
\text { baseline improve sign. during first } 6 \text { weeks } \\
(p=0.044) \text {, no diff at } 6 \text { mo }\end{array}$ & See Kendrick (2001) & / \\
\hline & & & $\begin{array}{l}\text { Diagnosis } \\
\text { sensitivity }\end{array}$ & No diff in sensitivity nor specificity & See Kendrick (2001) & / \\
\hline $\begin{array}{l}\text { King (2002) } \\
{[41]}\end{array}$ & $6 \mathrm{mo}$ & 10 & $\mathrm{BDI}$ & No sign. diff in BDI scores $(p=0.84)$ & $\begin{array}{l}\text { Smaller sample than anticipated } \\
\text { Cut off score for inclusion rather high } \\
\text { (makes intervention effect of CBT by lay } \\
\text { GPs less likely) }\end{array}$ & 0.08 \\
\hline $\begin{array}{l}\text { Llewellyn- } \\
\text { Jones } \\
\text { (1999) } \\
{[40]} \\
\end{array}$ & $9.5 \mathrm{mo}$ & 23 & GDS & Sign. change in GDS scores & $\begin{array}{l}\text { Serial mono-centered design } \\
\text { Control group assessment before } \\
\text { implementation of intervention }\end{array}$ & -0.17 \\
\hline $\begin{array}{l}\text { Rost (2001) } \\
{[44]}\end{array}$ & $6 \mathrm{mo}$ & 10 & mCES-D & $\begin{array}{l}\text { Sign. reduction in score in patients } \\
\text { beginning new treatment episode }\end{array}$ & $\begin{array}{l}\text { GP training effect unclear, feedback of } \\
\text { diagnosis could be responsible for } \\
\text { treatment effect } \\
\text { Homogenous sample }\end{array}$ & -0.29 \\
\hline $\begin{array}{l}\text { Pyne } \\
\text { (2003) [43] }\end{array}$ & $12 \mathrm{mo}$ & 65 & $\begin{array}{l}\text { Depression } \\
\text { severity }\end{array}$ & $\begin{array}{l}\text { Sign. decrease ( } 7.7 \text { units) in experimental } \\
\text { group }\end{array}$ & See Rost (2001) & / \\
\hline $\begin{array}{l}\text { Rost (2005) } \\
{[45]}\end{array}$ & $24 \mathrm{mo}$ & 70 & $\begin{array}{l}\text { Depression } \\
\text { Free Days }\end{array}$ & $\begin{array}{l}\text { Sign. more depression free days in } \\
\text { experimental group ( } 647.6 \text { vs. 558.2) }\end{array}$ & See Rost (2001) & / \\
\hline $\begin{array}{l}\text { Worrall } \\
\text { (1999) [39] }\end{array}$ & $6 \mathrm{mo}$ & $?$ & $\begin{array}{l}\text { Gain score } \\
\text { CES-D }\end{array}$ & Sign. improvement in experimental group & Possible Hawthorne effect & -0.22 \\
\hline
\end{tabular}

${ }^{a}$ all groups, last follow up; ${ }^{b}$ calculated as the mean difference of experimental and control group at the latest post-treatment measurement; ${ }^{\mathrm{c}}$ adopted from Bower et al. (2006) [45].

Abbreviations: BDI - Beck's Depression Inventory; CBT - Cognitive Behavioral Therapy; CE Ratio - Cost Effectiveness Ratio; mCES-D - (modified) Center for Epidemiologic Studies Depression Scale; CIDI - Composite International Diagnostic Interview; diff - difference; GDS - Geriatric Depression Scale; GP - General Practitioner; HAD - Hospital Anxiety and Depression Scale; HAM-D Hamilton Depression Scale; mo - month(s); MD - Major Depression; PRIME-MD - Primary care Evaluation of Mental Disorders; QALY - Quality of Adjusted Life Year; SF12 - Short Form 12-Item Health Survey; sign - significant 
elderly $(65+)$ adults in a residential facility, LlewellynJones and colleagues (1999) show a significant change in symptom quantity associated with a small effect size of Cohen's $\mathrm{d}=0.17$.

In studies that provided guidelines for the non-trained control group practitioners $[36,39,40]$, additional training and interventions in the experimental groups led to positive outcome changes (see tables 2 and 3 ).

\section{Meta-analysis}

The forest-plot of the conducted meta-analysis can be found in Figure 2. Three studies with only provider training provided data for meta-analysis. They found a non-significant decrease in depressive symptom load (pooled effect size $\mathrm{d}=-0.07,95 \%$ CI -0.24 to $0.10, \mathrm{I}^{2}=$ $21 \%)$.

Two studies were categorized as implementing additional guidelines to primary care. They showed the highest pooled effect sizes and a significant decrease in depressive symptoms in the intervention groups $(\mathrm{d}=-$ $0.26,95 \%$ CI -0.48 to -0.04$)$. The overall effect size, including a study with a more complex intervention was determined at $d=-0.15$ ( $95 \% \mathrm{CI}-0.27$ to -0.03$]$ ), favouring the experimental groups.

\section{Discussion}

It is apparent that there are only few trials evaluating the effect of primary care physician education on health outcomes of patients. Especially during the last six years no results of education based interventions have been published. It seems that the research focus has shifted to more complex interventions encompassing collaborative and stepped care approaches by introducing mental health specialists to the primary care setting [46-48]. Regarding the results of the reviewed studies, this approach seems more than justified - it has yet to be shown that training practitioners alone yields significant symptom changes; however, this conclusion is only based on three relevant studies that themselves are highly diverse. While the study by Gask et al. (2004) struggles with high attrition rates, King et al. (2002) used a rather high cut-off and included chronically depressed patients, possibly leading to a conservative bias and therefore underestimating the treatment effect $[38,41]$. The authors argue that the applied kind of brief cognitive behavioural therapy might have been treatment approach not sufficiently intense for highly depressed patients. Bosmans et al. (2006) find that including less severely affected patients might have led to an underestimation of the efficacy of anti-depressant treatment [33].

Sample selection plays a major role in assessing treatment effects in general. One could assume that severely affected patients benefit more from treatment in clinical studies (as they can show a higher reduction in quantity of symptoms). In line with this, a categorical diagnostic approach for patient inclusion by applying diagnostic categories as provided by the Diagnostic and Statistical Manual of Mental Disorders (DSM) might lead to a sample of more severely affected patients [33,36,37].

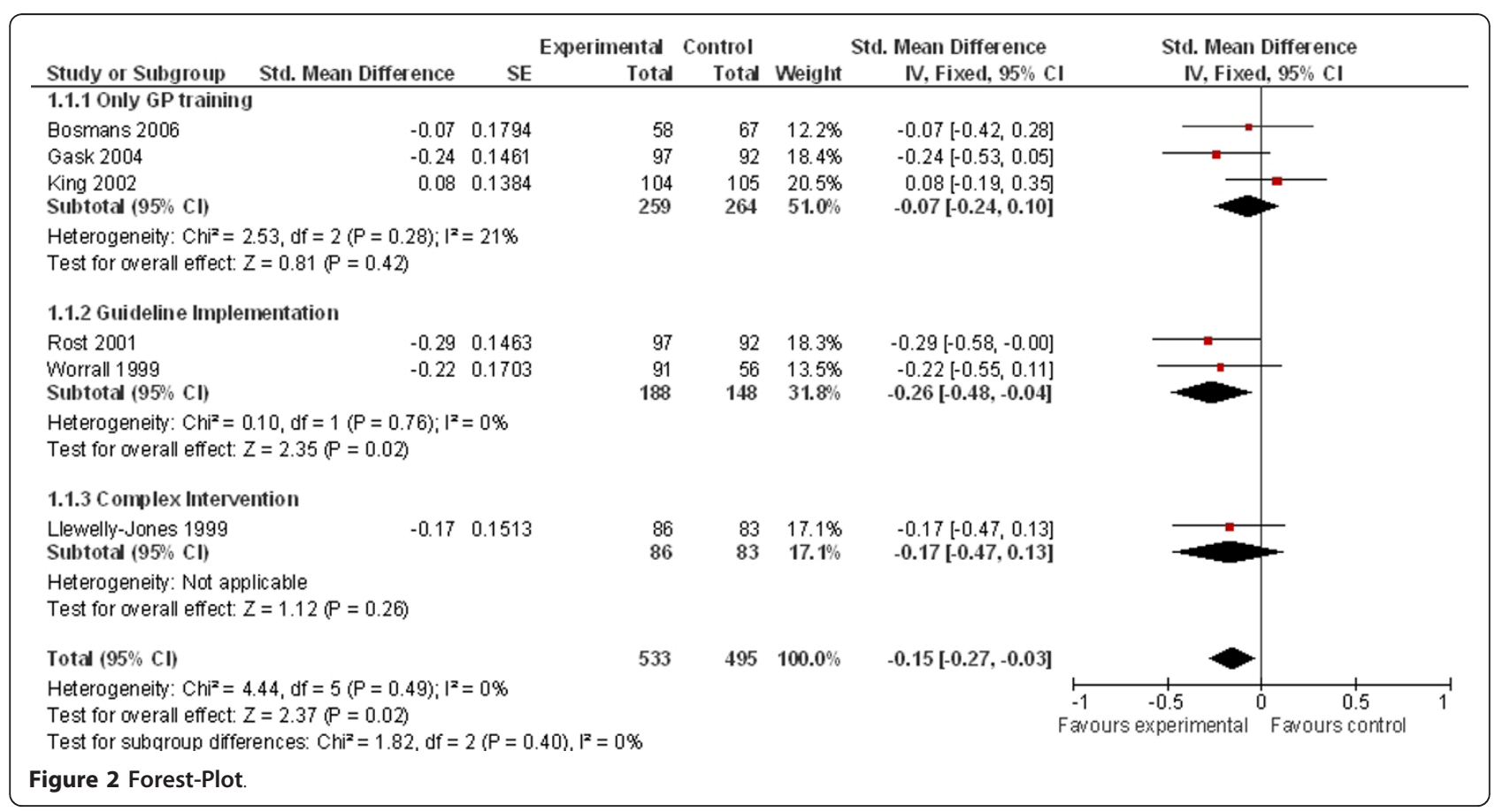


Furthermore, the kind of treatment has an effect. In the context of stepped care, this issue is addressed by providing more-intense treatment options to higher affected patients [25].

Both argumentations can provide explanations for the positive results found by studies implementing additional guideline usage by general practitioners. Small effect sizes were shown by those studies including patients with new-onset depression, rather than chronically depressed patients (as done by Kendrick et al., 2001 not resulting in positive symptom change). Obviously, the effect of mere attention to the trained practitioners as well as to the patients themselves (referred to as Hawthorne effect) has to be considered a possible moderating variable in study designs. This would lead to better outcomes and performances of the control groups even though they received no active intervention; thus, the differences found may possibly be even higher.

The justification for more complex interventions to improve primary care depression treatment is replicated in the analysis of included studies and basically goes in line with a previous review [21], however, we did find more evidence in newer studies that support guideline implementation to be effective in symptom reduction. One trial applying more complex strategies both yielded significant changes in symptom outcomes; however, it remains unclear how much of the effect can be attributed to the physicians' education. Bower et al. (2006) conducted a meta-regression to evaluate active ingredients of collaborative care interventions [49]. In this analysis, primary care physician training is not associated with a positive change in depressive symptoms nor with a change in antidepressant use even in univariate calculations. Rather than provider education, systematic identification of patients, professional background of staff and supervision proved to be significant predictors of symptom change. It becomes clear that researchers should not assume an additive effect of treatment modules; especially in view of economic considerations, collaborative care cannot mean implementing as many treatment options as possible. This analysis of one specific potentially effective part of collaborative care is leading the way to a more thorough understanding of complex interventions and has to be pursued without neglecting the fact that more simple interventions can also lead to significant changes in patient outcomes as shown in this review.

However, it may not be appropriate to solely focus on outcomes of symptomatology as enhanced primary care supply may not be directly associated with such. Even the included studies show a rise in adherence to medication treatment [39] and medication treatment in general $[34,44,50]$. It has been shown that effectiveness of antidepressant treatment increases with depression severity [51]; an effect of increased antidepressant treatment in a sample of mildly depressed patients will therefore be small [as seen in the studies by [33,39]].

\section{Strengths and Limitations}

This review only included randomised controlled trials, and therefore neglected observational and non-randomised studies. RCTs often adhere to strict exclusion criteria, thus making generalisability to primary care patients difficult. This also applies to the current review since studies with specialised co-morbid patient groups were excluded; however, regarding the heterogeneity of primary care patients, an adequate representation of studies seems hard to achieve in any case. The reported studies differ substantially in content, duration, intensity and frequency of the intervention programmes, making comparisons difficult. However, we were able to conduct a meta-analysis, quantifying the results of the studies. Meta-regression that could help determine the influence of these factors was not applicable due to the limited number of studies.

This partly results from the relatively narrow search strategy; only when education or training efforts of GPs were mentioned within title and abstract, the article was found with the applied search strategy. Earlier publications (before 1999) were not searched. Gilbody et al. mention one previous trial that showed positive effects of provider training but equally emphasise methodological weakness of this trial $[21,52]$, so we did include relevant trials that live up to methodological requirements.".

Furthermore, a possible publication bias cannot be ruled out or determined with a qualitative review as this, especially under the regard of not searching grey literature. Regarding the fact that almost only studies from anglophone countries were found might be an indicator for unpublished studies with negative outcomes from other countries.

\section{Conclusions}

It seems that provider training, if combined with guideline implementation, contributes to enhanced care for depression in primary care even associated with possible positive symptom changes. Providing a guideline and training practitioners to adhere to guideline-concordant treatment might be a measure of intervention that endures even after the intervention ends.

\section{Additional material} Additional file 1: Search terms for Medline. Details on the search
strategy for Medline.

Additional file 2: List and references of excluded studies. Overview of reason for exclusion. 


\section{Acknowledgement and funding}

This work is part of Esther-Net and was supported by the German Federal Ministry for Education and Research [grant number: 01 ET0719 (Esther-Net)]. The German Federal Ministry for Education and Research had no further role in the study design; in the collection, analyses and interpretation of data; in writing the report; and in the decision to submit the paper for publication. The publication of study results was not contingent on the sponsor's approval.

\section{Author details}

IInstitute of Social Medicine, Occupational Health and Public Health, University of Leipzig, Leipzig, Germany. ${ }^{2}$ IFB AdiposityDiseases, Leipzig University Medical Center, Leipzig, Germany. ${ }^{3}$ Department of Medical Sociology and Health Economics, Hamburg-Eppendorf University Medical Center, Hamburg, Germany. ${ }^{4}$ Department of Primary Medical Care, HamburgEppendorf University Medical Center, Hamburg, Germany. ${ }^{5}$ Institute of Social Medicine, Occupational Health and Public Health, University of Leipzig, Leipzig, Germany.

\section{Authors' contributions}

CS, ML and SRH outlined and specified the research questions. The principal author and ML conducted the literature search and screened abstracts and titles. Article inclusion and study quality was also evaluated by ML and SRH. CS wrote the first draft of the manuscript. HHK and HvdB revised it critically for important intellectual content. All authors contributed to and have approved the final manuscript.

\section{Competing interests}

The authors declare that they have no competing interests.

Received: 7 June 2011 Accepted: 10 January 2012

Published: 10 January 2012

\section{References}

1. Alonso J, Angermeyer MC, Bernert S, Bruffaerts R, Brugha TS, Bryson H, de Girolamo G, Graaf R, Demyttenaere K, Gasquet l, et al: Prevalence of mental disorders in Europe: results from the European Study of the Epidemiology of Mental Disorders (ESEMeD) project. Acta Psychiatr Scand Suppl 2004, 21-27.

2. Wittchen HU, Jacobi F: Size and burden of mental disorders in Europe-a critical review and appraisal of 27 studies. Eur Neuropsychopharmacol 2005, 15:357-376.

3. Frei A, Ajdacic-Gross V, Rossler W, Eich-Hochli D: Effects of depressive disorders on objective life quality criteria. Psychiatr Prax 2004, 31:298-303.

4. Greer TL, Kurian BT, Trivedi MH: Defining and measuring functional recovery from depression. CNS Drugs 2010, 24:267-284.

5. Cuijpers P, Smit F: Excess mortality in depression: a meta-analysis of community studies. J Affect Disord 2002, 72:227-236.

6. Luppa M, Heinrich S, Angermeyer MC, König HH, Riedel-Heller SG: Cost-ofillness studies of depression: a systematic review. J Affect Disord 2007, 98:29-43.

7. Gunther $\mathrm{OH}$, Friemel $\mathrm{S}$, Bernert $\mathrm{S}$, Matschinger $\mathrm{H}$, Angermeyer MC, König HH: [The burden of depressive disorders in Germany - results from the European Study of the Epidemiology of Mental Disorders (ESEMeD)]. Psychiatr Prax 2007, 34:292-301.

8. Kessler RC, Heeringa S, Lakoma MD, Petukhova M, Rupp AE, Schoenbaum M, Wang PS, Zaslavsky AM: Individual and societal effects of mental disorders on earnings in the United States: results from the national comorbidity survey replication. Am J Psychiatry 2008, 165:703-711.

9. König HH, Luppa M, Riedel-Heller SG: [The Costs of Depression and the Cost-Effectiveness of Treatment]. Psychiatr Prax 2010, 37:213-215.

10. Halfin A: Depression: the benefits of early and appropriate treatment. Am J Manag Care 2007, 13:592-597.

11. Agency for Healthcare Research and Quality: Improving quality of care for people with depression. Translating research into practice. Fact sheet. 2000 [http://www.ahrq.gov/research/deprqoc.htm], accessed 1-7-2011.

12. Wittchen HU, Holsboer F, Jacobi F: Met and unmet needs in the management of depressive disorder in the community and primary care: the size and breadth of the problem. J Clin Psychiatry 2001, 62(Suppl 26):23-28.
13. Bijl D, van Marwijk HW, Haan M, van Tilburg W, Beekman AJ: Effectiveness of disease management programmes for recognition, diagnosis and treatment of depression in primary care. Eur J Gen Pract 2004, 10:6-12.

14. Dowrick C: Does testing for depression influence diagnosis or management by general practitioners? Fam Pract 1995, 12:461-465.

15. Dowrick C, Buchan I: Twelve month outcome of depression in general practice: does detection or disclosure make a difference? BMJ 1995, 311:1274-1276.

16. Sielk M, Altiner A, Janssen B, Becker N, de Pilars MP, Abholz HH: [Prevalence and diagnosis of depression in primary care. A critical comparison between PHQ-9 and GPs' judgement]. Psychiatr Prax 2009, 36:169-174.

17. Spitzer RL, Wakefield JC: DSM-IV diagnostic criterion for clinical significance: does it help solve the false positives problem? Am J Psychiatry 1999, 156:1856-1864.

18. Prins MA, Verhaak PF, van der MK, Penninx BW, Bensing JM: Primary care patients with anxiety and depression: need for care from the patient's perspective. J Affect Disord 2009, 119:163-171.

19. U.S. Preventive Services Task Force: Screening for depression in adults: U. S. preventive services task force recommendation statement. Ann Int Med 2009, 151:784-792.

20. Gilbody S, House A, Sheldon T: Screening and case finding instruments for depression. Cochrane Database of Systematic Reviews 2005.

21. Gilbody S, Whitty P, Grimshaw J, Thomas R: Educational and organizational interventions to improve the management of depression in primary care: a systematic review. JAMA 2003, 289:3145-3151.

22. Huibers MJH, Beurskens A, Bleijenberg G, van Schayck CP: Psychosocial interventions by general practitioners. Cochrane Database of Systematic Reviews 2007.

23. MCNaughton JL: Brief interventions for depression in primary care: a systematic review. Can Fam Physician 2009, 55:789-796.

24. Schlette S, Lisac M, Blum K: Integrated primary care in Germany: the road ahead. Int J Integr Care 2009, 9:e14.

25. National Institute for Health and Clinical Excellence: Depression in Adults. National Clinical Practice Guideline 90 London: NICE; 2009.

26. Patten $\mathrm{SB}$, Bilsker $\mathrm{D}$, Goldner $\mathrm{E}$ : The evolving understanding of major depression epidemiology: implications for practice and policy. Can J Psychiatry 2008, 53:689-695.

27. Cape J, Whittington C, Bower P: What is the role of consultation-liaison psychiatry in the management of depression in primary care? A systematic review and meta-analysis. Gen Hosp Psychiatry 2010, 32:246-254.

28. Centre for Reviews and Dissemination: Systematic Review. CDR's guidance for undertaking reviews in health care. 2009 [http://www.yps-publishing. co.uk], accessed 1-7-2011.

29. Moher D, Liberati A, Tetzlaff J, Altman DG: Preferred reporting items for systematic reviews and meta-analyses: the PRISMA statement. J Clin Epidemiol 2009, 62:1006-1012.

30. Moncrieff J, Churchill R, Drummond DC, McGuire H: Development of a quality assessment instrument for trials of treatments for depression and neurosis. Int J Methods Psychiatr Res 2001, 10:126-133.

31. Cohen JW: Statistical power analysis for the behavioral sciences Hillsdale, NJ: Erlbaum; 1988.

32. Review Manager (RevMan). Copenhagen, The Nordic Cochrane Centre, The Cochrane Collaboration; 2011, (Version 5.1).

33. Bosmans J, De Bruijne M, Van Hout $H$, Van Marwijk H, Beekman A, Bouter L, Stalman W, Van Tulder M: Cost-Effectiveness of a Disease Management Program for Major Depression in Elderly Primary Care Patients. J Gen Intern Med 2006, 21:1020-1026

34. Kendrick T, Stevens L, Bryant A, Goddard J, Stevens A, Raftery J, Thompson C: Hampshire depression project: changes in the process of care and cost consequences. Br J Gen Pract 2001, 51:911-913.

35. Thompson C, Kinmonth AL, Stevens L, Peveler RC, Stevens A, Ostler KJ, Pickering RM, Baker NG, Henson A, Preece J, et al: Effects of a clinicalpractice guideline and practice-based education on detection and outcome of depression in primary care: Hampshire Depression Project randomised controlled trial. Lancet 2000, 355:185-191.

36. Baker R, Reddish S, Robertson N, Hearnshaw H, Jones B: Randomised controlled trial of tailored strategies to implement guidelines for the management of patients with depression in general practice. $\mathrm{Br} J \mathrm{Gen}$ Pract 2001, 51:737-741. 
37. Rost K, Nutting PA, Smith J, Werner JJ: Designing and implementing a primary care intervention trial to improve the quality and outcome of care for major depression. Gen Hosp Psychiatry 2000, 22:66-77.

38. Gask L, Dowrick C, Dixon C, Sutton C, Perry R, Torgerson D, Usherwood T: A pragmatic cluster randomized controlled trial of an educational intervention for GPs in the assessment and management of depression. Psychol Med 2004, 34:63-72.

39. Worrall G, Angel J, Chaulk P, Clarke C, Robbins M: Effectiveness of an educational strategy to improve family physicians' detection and management of depression: a randomized controlled trial. CMAJ 1999, 161:37-40.

40. Llewellyn-Jones RH, Baikie KA, Smithers H, Cohen J, Snowdon J, Tennant CC: Multifaceted shared care intervention for late life depression in residential care: randomised controlled trial. BMJ 1999, 319:676-682

41. King M, Davidson O, Taylor F, Haines A, Sharp D, Turner R: Effectiveness of teaching general practitioners skills in brief cognitive behaviour therapy to treat patients with depression: randomised controlled trial. BMJ 2002, 324:947-950

42. Higgins JPT, Green S: Cochrane Handbook for Systematic Reviews of Interventions Chichester: Wiley; 2008

43. Pyne JM, Rost KM, Zhang M, Williams DK, Smith J, Fortney J: Costeffectiveness of a primary care depression intervention. J Gen Intern Med 2003, 18:432-441.

44. Rost K, Nutting P, Smith J, Werner J, Duan N: Improving depression outcomes in community primary care practice: a randomized trial of the quEST intervention. Quality Enhancement by Strategic Teaming. J Gen Intern Med 2001, 16:143-149.

45. Rost K, Pyne JM, Dickinson LM, LoSasso AT: Cost-effectiveness of enhancing primary care depression management on an ongoing basis. Ann Fam Med 2005, 3:7-14.

46. Bower PJ, Rowland N: Effectiveness and cost effectiveness of counselling in primary care. Cochrane Database of Systematic Reviews 2006.

47. Skultety KM, Zeiss A: The treatment of depression in older adults in the primary care setting: an evidence-based review. Health Psychol 2006, 25:665-674.

48. Skultety KM, Rodriguez RL: Treating geriatric depression in primary care. Curr Psychiatry Rep 2008, 10:44-50.

49. Bower PJ, Gilbody S, Richards D, Fletcher J, Sutton A: Collaborative care for depression in primary care. Making sense of a complex intervention: systematic review and meta-regression. Br J Psychiatry 2006, 189:484-493.

50. Roskar S, Podlesek A, Zorko M, Tavcar R, Dernovsek MZ, Groleger U, Mirjanic $M$, Konec $N$, Janet $E$, Marusic $A$ : Effects of training program on recognition and management of depression and suicide risk evaluation for Slovenian primary-care physicians: follow-up study. Croat Med J 2010, 51:237-242.

51. Fournier JC, Derubeis RJ, Hollon SD, Dimidjian S, Amsterdam JD, Shelton RC, Fawcett J: Antidepressant drug effects and depression severity: a patient-level meta-analysis. JAMA 2010, 303:47-53.

52. Rutz W, von Knorring L, Walinder J: Frequency of suicide on Gotland after systematic postgraduate education of general practitioners. Acta Psychiatr Scand 1989, 80:151-154.

Pre-publication history

The pre-publication history for this paper can be accessed here: http://www.biomedcentral.com/1472-6963/12/10/prepub

doi:10.1186/1472-6963-12-10

Cite this article as: Sikorski et al:: Does GP training in depression care affect patient outcome? - A systematic review and meta-analysis. BMC Health Services Research 2012 12:10.

\section{Submit your next manuscript to BioMed Central and take full advantage of:}

- Convenient online submission

- Thorough peer review

- No space constraints or color figure charges

- Immediate publication on acceptance

- Inclusion in PubMed, CAS, Scopus and Google Scholar

- Research which is freely available for redistribution

Submit your manuscript a www.biomedcentral.com/submit
C) Biomed Central 